\title{
Ozonização em câmara fria durante a maturação parcial de queijo parmesão
}

\author{
Ozonation in a cold room during the partial maturation of parmesan cheese \\ Ozonización en cámara frigorífica durante la maduración parcial del queso parmesano
}

Recebido: 03/12/2021 | Revisado: 11/12/2021 | Aceito: 14/12/2021 | Publicado: 21/12/2021

\author{
Igor Moura Gargaro \\ ORCID: https://orcid.org/0000-0001-7023-8266 \\ Universidade Estadual de Maringá, Brasil \\ E-mail: igorgargaro@gmail.com \\ Mayara Garcete Baldacim \\ ORCID: https://orcid.org/0000-0002-0473-252X \\ Universidade Estadual de Maringá, Brasil \\ E-mail: mayaragaba@gmail.com \\ Annecler Rech de Marins \\ ORCID: https://orcid.org/0000-0001-8395-6726 \\ Universidade Estadual de Maringá, Brasil \\ E-mail: anneclermarins@ gmail.com \\ Talita Aparecida Ferreira de Campos \\ ORCID: https://orcid.org/0000-0003-2236-3913 \\ E-mail: Talita.tc29@gmail.com \\ Universidade Estadual de Maringá, Brasil \\ Natallya Marques da Silva \\ ORCID: https://orcid.org/0000-0001-5273-3749 \\ Universidade Estadual de Maringá, Brasil \\ E-mail: natallyaengali@outlook.com \\ Andresa Carla Feihrmann \\ ORCID: https://orcid.org/0000-0003-2389-0467 \\ Universidade Estadual de Maringá, Brasil \\ E-mail: andresafeihrmann@gmail.com
}

\begin{abstract}
Resumo
Durante a produção de queijo pode ocorrer a contaminação microbiana, sendo a falta de higiene comum contribuinte para a contaminação. As câmeras de maturação de queijos também se destacam no favorecimento da multiplicação fúngica, devido às suas condições de temperatura e umidade, sendo o crescimento de fungos no queijo tipo parmesão prejudicial tanto a sua composição quanto sua estrutura e aparência, o que pode ocasionar a rejeição pelo consumidor, causando perda econômica além do desperdício de matéria-prima. Assim o ozônio foi utilizado na maturação de queijo parmesão, com o objetivo de controlar o crescimento fúngico. Foram realizadas análises de atividade de água, cor e análises microbiológicas durante 90 dias. O gás não apresentou influência na atividade de água dos queijos, já nos resultados de cor constatou-se que não houve diferença significativa apenas para as medições de 90 dias. Para os resultados de coliformes a 35 e $45^{\circ} \mathrm{C}$, estafilococos e Salmonella, foram encontrados valores que atendiam as especificações. Com relação aos resultados de bolores e leveduras ozônio conseguiu reduzir a contagem no ambiente de maturação.
\end{abstract}

Palavras-chave: Queijo tipo parmesão; Maturação; Ozônio; Crescimento fúngico.

\begin{abstract}
Microbial contamination can occur during cheese production, and lack of common hygiene contributes to the contamination. Cheese maturation chambers also stand out in favoring fungal multiplication, due to their temperature and humidity conditions, the growth of fungi in parmesan cheese being harmful to both its composition and its structure and appearance, which can lead to rejection by the consumer, causing economic loss in addition to the waste of raw materials. Thus, ozone was used in the maturation of Parmesan cheese, in order to control fungal growth. Analyzes of water activity, color and microbiological analyzes were carried out for 90 days. The gas had no influence on the water activity of the cheeses, whereas in the color results it was found that there was no significant difference only for the 90 day measurements. For the results of coliforms at 35 and $45^{\circ} \mathrm{C}$, staphylococci and Salmonella, values were found that met the specifications. Regarding the results of molds and yeasts, ozone managed to reduce the count in the ripening environment.
\end{abstract}

Keywords: Parmesan type cheese; Maturation; Ozone; Fungal growth.

\section{Resumen}

La contaminación microbiana puede ocurrir durante la producción de queso y la falta de higiene común contribuye a la contaminación. Las cámaras de maduración del queso también destacan por favorecer la multiplicación de hongos, por 
sus condiciones de temperatura y humedad, siendo el crecimiento de hongos en el queso parmesano perjudicial tanto para su composición como para su estructura y apariencia, lo que puede llevar al rechazo por parte del consumidor, provocando pérdidas económicas. además del desperdicio de materias primas. Por lo tanto, se utilizó ozono en la maduración del queso parmesano, con el fin de controlar el crecimiento de hongos. Se realizaron análisis de la actividad del agua, color y análisis microbiológicos durante 90 días. El gas no tuvo influencia sobre la actividad del agua de los quesos, mientras que en los resultados de color se encontró que no hubo diferencia significativa solo para las mediciones de 90 días. Para los resultados de coliformes a 35 y $45^{\circ} \mathrm{C}$, estafilococos y Salmonella, se encontraron valores que cumplieron con las especificaciones. En cuanto a los resultados de mohos y levaduras, el ozono logró reducir el recuento en el ambiente de maduración.

Palabras clave: Queso tipo parmesano; Maduración; Ozono; Crecimiento de hongos.

\section{Introdução}

O queijo parmesão também conhecido como Parmigiano-Reggiano, Grana ou Grana Padano, é produzido em uma área muito restrita que está localizada quase que completamente na região de Emilia-Romagna (Itália central) e sua maturação normalmente acontece entre um a três anos, até que se torne macio e quebradiço (Argenti et al., 2021; Sora et al., 2013). Sendo muito apreciado pelos consumidores em todo o mundo, devido ao seu sabor característico, centrado em torno de umami, salgado, amargo-doce, notas ardentes, e seu valor nutritivo (Hillmann \& Hofmann, 2016). No Brasil o queijo parmesão ralado é um produto obtido a partir do esfarelamento ou ralagem da massa de uma ou até quatro variedades, sendo normalmente consumido com molhos e massas (Pimentel et al., 2002; Trombete et al., 2013). A maturação do queijo parmesão no Brasil acontece em seis meses enquanto o queijo tipo Grana possui um período de maturação de 12 meses (Sora et al., 2013).

Durante a produção de queijo pode ocorrer a contaminação microbiana, sendo a falta de higiene e/ou falta de controle comuns contribuintes para a contaminação (Fernandes et al., 2018). Além disso, outros fatores se destacam podendo tornar ambiente favorável ao crescimento de microrganismos, como o pH, a concentração de sal, temperatura, atividade de água, tempo de amadurecimento e temperatura de armazenamento do queijo (Custódio et al., 2007). As câmeras de maturação de queijos também se destacam no favorecimento da multiplicação fúngica, devido às suas condições de temperatura e umidade, sendo os fungos ambientais indesejáveis (Pinto et al., 2007), uma vez que algumas espécies são produtoras de micotoxinas (Franco \& Landgraf, 2005) e o seu crescimento no queijo tipo parmesão pode prejudicar tanto a sua composição quanto sua estrutura e aparência, o que pode ocasionar a rejeição do produto pelo consumidor, causando perda econômica além do desperdício de matéria-prima (Lanita \& Silva, 2008).

Algumas estratégias têm sido adotadas para controlar o crescimento fúngico na superfície dos queijos e no ar da câmara de maturação nas indústrias, porém nem sempre elas são eficientes economicamente e ambientalmente, além da possibilidade de permanecerem no produto resíduos de compostos coadjuvantes de tecnologia utilizados (Lanita \& Silva, 2008). O ozônio é um gás azulado com odor pungente, obtido quando as moléculas de oxigênio são submetidas à descarga elétrica de alta voltagem e ocorre o rearranjo de átomos, sendo um potente agente antimicrobiano que pode ser usado contra bactérias, fungos, vírus, protozoários e esporos (Horvath et al., 1985; Khadre et al., 2001). Seu mecanismo de ação consiste na lise celular, onde os microrganismos são erradicados pela ruptura ou degradação do envelope celular, sendo considerado um método mais rápido do que o de outros agentes antimicrobianos (Srey et al., 2013).

Assim objetivou-se nesse estudo utilizar o ozônio na maturação de queijo parmesão e realizar análises de atividade de água, cor e análises microbiológicas durante 90 dias, afim de observar o crescimento fúngico.

\section{Material e Métodos}

\subsection{Materiais}

Esta pesquisa é caracterizada como experimental (Pereira et al., 2018). O queijo parmesão foi fornecido por uma empresa localizada no interior do estado de São Paulo. A peça de queijo possuía em média $8 \mathrm{~kg}$ e foi dividida em 8 pedaços de $1 \mathrm{~kg}$ cada. 
Posteriormente, as porções foram expostas nas prateleiras da câmara fria, sem ozonização $\left(404 \mathrm{~m}^{3}\right)$ e na câmara fria com ozonização $\left(870 \mathrm{~m}^{3}\right)$. O gerador de ozônio utilizado (Interozone) era do modelo TES - 485 R33 com 465 watts de potência nominal e sua capacidade de geração de ozônio podia ser realizada em níveis de 1 a 3, sendo o 3 o mais alto nível. A temperatura das câmaras foi mantida entre $10-12^{\circ} \mathrm{C}$. Para a execução das análises os queijos sem exposição ao ozônio (QP) e os expostos ao ozônio (QPO) foram coletados na empresa, acondicionados e transportados em isopor até o laboratório onde foram mantidos em geladeira a $10^{\circ} \mathrm{C}$ até a realização das análises.

\subsection{Atividade de água (Aw)}

A atividade de água foi determinada em temperatura ambiente, $25^{\circ} \mathrm{C}$, utilizando o medidor de atividade de água marca Aw Sprint - Novasina TH-50. A medição foi realizada em triplicata nos dias 0, 45 e 90.

\subsection{Cor instrumental}

Para determinação da cor foi utilizado o colorímetro CR-400 Minolta Chromameter (Minolta Cia Ltda.), no espaço CIE $\mathrm{L}^{*} \mathrm{a}^{*} \mathrm{~b}^{*}$, onde $\mathrm{L}^{*}=$ luminosidade, que varia de zero sendo o preto e 100 sendo o branco, $\mathrm{a}^{*}=$ intensidade da cor vermelha variando de -80 até zero sendo verde e de zero até 100 sendo a cor vermelha, e $b^{*}=$ intensidade da cor amarela, onde de -100 até zero sendo a cor azul e do zero ao 70 sendo a cor amarela. O equipamento foi calibrado utilizando o branco, com iluminante D65, $10^{\circ}$ observador padrão e tamanho de abertura de $8 \mathrm{~mm}$ de diâmetro. As medidas foram feitas em triplicata nos dias 0,45 e 90.

\subsection{Análises microbiológicas}

As análises microbiológicas para coliformes a $35^{\circ} \mathrm{C}$ e a $45^{\circ} \mathrm{C}$, Salmonella spp., Staphylococcus aureus coagulase positiva e bolores e leveduras foram realizadas de acordo com APHA (2001). As análises foram realizadas nos dias 0, 45 e 90, em triplicata. Os resultados foram expressos em número mais provável (NMP) para coliformes a $35{ }^{\circ} \mathrm{C}$ e a $45{ }^{\circ} \mathrm{C}$ e em unidades formadoras de colônias por grama (UFC/g) para as demais análises.

\subsection{Análise estatística}

Para a análise estatística foi utilizado o programa SASM- Agri: Sistema para análise e separação de médias em experimentos agrícolas pelos métodos Scoft - Knott, Tukey e Duncan (Canteri et al., 2001). Foi realizada a análise de variância (ANOVA) e aplicado o teste de Tukey para comparação das médias das amostras, considerando um nível de significância de $95 \%$.

\section{Resultados e Discussão}

\subsection{Atividade de água}

Os resultados da atividade de água do queijo parmesão submetido ou não ao ozônio são apresentados na Tabela 1. O gás não apresentou influência na atividade de água dos queijos, porém no dia 90 os tratamentos apresentaram os maiores valores e diferiram significativamente $(\mathrm{P}<0.05)$ dos dias 0 e 45. A amostra QP apresentou valor de 0,965 enquanto a amostra QPO apresentou valor de 0,956 ao final da maturação. Segundo Barros et al., (2011) a atividade de água em queijos parmesão com 180 dias de cura variou de $0,975 \pm 0,01$ a $0,979 \pm 0,01$, valores mais altos ao desse estudo, porém a maturação não completou os 180 dias. 
Tabela 1. Influência da exposição do queijo parmesão ao gás ozônio na atividade de água.

\begin{tabular}{llcc}
\hline & & Tratamentos & \\
\cline { 3 - 4 } & & QP & QPO \\
\hline \multirow{3}{*}{ Atividade de água } & Dia 0 & $0,904 \pm 0,009^{\mathrm{b}}$ & $0,904 \pm 0,009^{\mathrm{b}}$ \\
& Dia 45 & $0,893 \pm 0,004^{\mathrm{b}}$ & $0,895 \pm 0,004^{\mathrm{b}}$ \\
& Dia 90 & $0,956 \pm 0,002^{\mathrm{a}}$ & $0,965 \pm 0,004^{\mathrm{a}}$ \\
\hline
\end{tabular}

${ }^{a-b}$ Médias seguidas de letras minúsculas diferentes na mesma coluna diferem estatisticamente pelo teste de Tukey ao nível de 5\% de significância. QP: sem exposição ao ozônio. QPO: com exposição ao ozônio. Fonte: Autores

Quando comparado ao estudo de Starikoff et al. (2016), que avaliaram a dimunuição dos microrganismos Mycobacterium bovis e da Brucella abortus durante a cura de queijo tipo parmesão, o comportamento de diminuição da atividade de água ao longo da maturação não foi observado nesse estudo e os valores finais foram menores que os de queijo parmesão contaminado experimentalmente $(0,976)$. Embalagens termoencolhíveis não foram aplicadas, elas possuem o intuito de evitar perda excessiva de água e rachaduras na superfície, o que justifica os valores mais baixos de Aw (Barros et al., 2011). Apesar disso, segundo Jaster et al. (2014) uma baixa atividade de água faz parte das características médias esperadas para o queijo do tipo parmesão.

\subsection{Cor instrumental}

A Tabela 2 indica os resultados obtidos da análise instrumental da cor do queijo. Cada medida foi feita em triplicata, da superfície, interior e um ponto entre a superfície e interior do queijo. Quanto a luminosidade os tratamentos aos 45 dias de maturação apresentaram diferença significativa entre si e diferença significativa $(\mathrm{P}<0.05)$ com os demais tempos, porém as amostras com 90 dias de fabricação não apresentaram diferença entre si. QP e QPO apresentaram valores, no dia 90, de 64,8 e 63,4 de luminosidade respectivamente, sendo menores quando comparado com a luminosidade relatada por Kędzierska-Matysek et al. (2018) (76,08-76,99) ao avaliarem as propriedades físico-químicas, mecânicas e sensoriais de queijo Parmigiano Reggiano e Grana Padano já amadurecidos. O maior valor de $L^{*}$ no dia 0 (73,3), condiz com o fato do queijo estar sem nenhuma maturação, assim não desenvolveu o amarelo escuro característico do queijo parmesão, apresentando um amarelo ligeiramente claro.

Tabela 2. Influência da exposição do queijo parmesão ao gás ozônio nos parâmetros de cor.

\begin{tabular}{llccc}
\hline & \multicolumn{4}{c}{ Parâmetros cor } \\
\cline { 3 - 5 } & Dia 0 & $73,303 \pm 1,32^{\mathrm{a}}$ & $-7,630 \pm 0,16^{\mathrm{c}}$ & $10,853 \pm 0,35^{\mathrm{d}}$ \\
\hline \multirow{2}{*}{ QP } & Dia 45 & $45,280 \pm 2,24^{\mathrm{d}}$ & $-5,756 \pm 0,32^{\mathrm{a}}$ & $16,003 \pm 0,88^{\mathrm{c}}$ \\
& Dia 90 & $64,880 \pm 1,04^{\mathrm{b}}$ & $-8,376 \pm 0,13^{\mathrm{d}}$ & $25,376 \pm 0,69^{\mathrm{a}}$ \\
\multirow{2}{*}{ QPO } & Dia 45 & $58,463 \pm 0,28^{\mathrm{c}}$ & $-6,503 \pm 0,08^{\mathrm{b}}$ & $18,886 \pm 0,17^{\mathrm{b}}$ \\
& Dia 90 & $63,446 \pm 0,43^{\mathrm{b}}$ & $-8,166 \pm 0,12^{\mathrm{d}}$ & $24,546 \pm 0,70^{\mathrm{a}}$ \\
\hline
\end{tabular}

${ }^{\text {a-d }}$ Médias seguidas de letras minúsculas diferentes na mesma coluna diferem estatisticamente pelo teste de Tukey ao nível de 5\% de significância. QP: sem exposição ao ozônio. QPO: com exposição ao ozônio. Fonte: Autores

Em relação aos valores do parâmetro a* que varia da cor verde (-) a cor vermelha (+), percebeu-se que as amostras se encontram dentro do espectro da coloração verde (-). Não houve diferença significativa entre QP e QPO com 90 dias de maturação, apresentando valores menores e significativamente diferentes quando comparados com os outros dias de maturação.

O parâmetro b* varia da cor azul (-) a cor amarela (+), os resultados desse parâmetro encontrados mostram que o queijo está dentro do espectro amarelo, e que com o passar dos dias de maturação a cor amarela for intensificada. As amostras com 90 dias de maturação não apresentaram diferença significativa entre si, e as demais amostras apresentaram valores significativamente menores, sendo que a formulação QPO aos 45 dias possuía uma coloração mais amarelada do que a 
formulação QP nesse mesmo tempo. Quando comparados esses valores, os queijos desse estudo apresentam uma coloração amarela mais escura que queijos Parmigiano Reggiano e Grana Padano já maturados (14,68-20,74).

\subsection{Análises microbiológicas}

A Todas as amostras foram testadas quanto à presença ou ausência de Salmonella sp e Staphylococcus coagulase positiva, e todos os tratamentos apresentaram ausência das mesmas. Com relação a coliformes a $35^{\circ} \mathrm{C}$ e a $45^{\circ} \mathrm{C}$ os resultados foram menores que 3. Esses resultados evidenciam que a produção dos queijos foi adequada, atendendo aos padrões de qualidade e segurança, sendo que os produtos não tiveram contaminação por matéria oriunda de origem fecal.

A Tabela 3 apresenta os resultados em UFC/g obtidos na análise de bolores e leveduras. As contagens oscilaram entre $1,2 \times 10^{3}$ e $>10^{5}$.

Tabela 3. Contagem de bolores e leveduras em queijo submetido ou não ao gás ozônio.

\begin{tabular}{|c|c|c|}
\hline & & Bolores e leveduras \\
\hline & Dia 0 & $1,2 \times 10^{3}$ \\
\hline \multirow{2}{*}{ QP } & Dia 45 & $>10^{5}$ \\
\hline & Dia 90 & $9,0 \times 10^{5}$ \\
\hline \multirow{2}{*}{ QPO } & Dia 45 & $1,3 \times 10^{3}$ \\
\hline & Dia 90 & $2,0 \times 10^{3}$ \\
\hline
\end{tabular}

Fonte: Autores.

As formulações submetidas ao gás ozônio apresentaram contagens mais baixas do que os produtos que não tiveram contato com o gás durante a maturação. Comparando os resultados, nos dois tempos, de QPO com o dia 0 pode-se observar que não houve um grande aumento na contagem, enquanto QP apresentou valor > 105 para o dia 45 e no dia 90 uma contagem elevada (9,0x105) se comparado com QPO. Outros estudos também observaram essa diminuição na contagem ao realizar a aplicação do ozônio na câmera de maturação (Lanita \& Silva, 2008; Pinto et al., 2007). Com a utilização do ozônio na câmara de maturação, tem-se a possibilidade de redução da mão de obra operacional, de redução no consumo de água (uma vez que são necessárias menos lavagens) e de eliminação do conservante natamicina do processo (Lanita \& Silva, 2008). O uso do ozônio está associado a obtenção de um produto com uma maior segurança alimentar e com maior vida de prateleira (Khadre et al., 2001; Kim et al., 1999).

\section{Conclusão}

Os resultados obtidos neste trabalho mostraram que o ozônio conseguiu reduzir a contagem de bolores e leveduras no ambiente de maturação. Sendo que o uso do ozônio não eliminou a proliferação, mas retardou o seu crescimento, o que é um efeito considerável na maturação de queijos. Além disso, a cor não foi afetada ao final da maturação pela utilização do ozônio. Sugere-se que estudos futuros avaliem técnicas e coadjuvantes de origem natural para eliminar a proliferação de bolores e leveduras.

\section{Referências}

APHA, 2001. Frances, P.D., Keith, I. (Eds.), Compendium of Methods for the Microbiological Examination of Foods (4th ed), American Public Health Association, Washington, DC.

Argenti, G., Parrini, S., Staglianò, N., \& Bozzi, R. (2021). Evolution of production and forage quality in sown meadows of a mountain area inside parmesan cheese consortium. Agronomy Research, 19(2), 344-356. https://doi.org/10.15159/AR.21.061

Barros, J. J. de C., Azevedo, A. C. de, Faleiros Júnior, L. R., Taboga, S. R., \& Penna, A. L. B. (2011). Queijo Parmesão: caracterização físico-química, microbiológica e microestrutura. Ciência e Tecnologia de Alimentos, 31(2), 285-294. https://doi.org/10.1590/S0101-20612011000200002 
Canteri, M. G., Althaus, R. A., Virgens Filho, J. S. Giglioti, E. A., \& Godoy, C. V. (2001). Sistema para análise e separação de médias em experimentos agrícolas pelos métodos Scoft - Knott, Tukey e Duncan. Revista Brasileira de Agrocomputação, 1(2), $18-24$.

Custódio, F. B., Tavares, É., \& Glória, M. B. A. (2007). Extraction of bioactive amines from grated Parmesan cheese using acid, alkaline and organic solvents. Journal of Food Composition and Analysis, 20(3-4), 280-288. https://doi.org/10.1016/j.jfca.2006.06.009

Fernandes, R. V. B., Botrel, D. A., Monteiro, P. S., Borges, S. V., Souza, A. U., \& Mendes, L. E. S. (2018). Microencapsulated oregano essential oil in grated Parmesan cheese conservation. International Food Research Journal, 25(2), 661-669.

Franco, B. D. G. M., \& Landgraf, M. (2005). Microbiologia dos Alimentos (2nd ed.). Atheneu.

Hillmann, H., \& Hofmann, T. (2016). Quantitation of Key Tastants and Re-engineering the Taste of Parmesan Cheese. Journal of Agricultural and Food Chemistry, 64(8), 1794-1805. https://doi.org/10.1021/acs.jafc.6b00112

Horvath, M., Bilitzky, L., \& Huttner, J. (1985). Ozone. Elsevier.

Jaster, H., de Campos, A. C. L. P., Auer, L. B., Los, F. G. B., Salem, R. D. S., Esmerino, L. A., Nogueira, A., \& Demiate, I. M. (2014). Quality evaluation of parmesan-type cheese: A chemometric approach. Food Science and Technology, 34(1), 181-188. https://doi.org/10.1590/S0101-20612014000100026

Kędzierska-Matysek, M., Barłowska, J., Wolanciuk, A., \& Litwińczuk, Z. (2018). Physicochemical, mechanical and sensory properties of long-ripened Polish and Italian cheeses and their content of selected minerals. Journal of Elementology, 23(3), 985-998. https://doi.org/10.5601/jelem.2017.22.4.1452

Khadre, M. A., Yousef, A. E., \& Kim, J. G. (2001). Microbiological aspects of ozone applications in food: A review. Journal of Food Science, 66(9), 12421252. https://doi.org/10.1111/j.1365-2621.2001.tb15196.x

Kim, J. G., Yousef, A. E., \& Dave, S. (1999). Application of ozone for enhancing the microbiological safety and quality of foods: A review. Journal of Food Protection, 62(9), 1071-1087. https://doi.org/10.4315/0362-028X-62.9.1071

Lanita, C. de S., \& Silva, S. B. (2008). Uso de ozônio em câmara industrial para controle de bolores e leveduras durante a maturação de queijo tipo parmesão. Brazilian Journal of Food Technology, 11, 182-189.

Pereira, S. A., Shitsuka, D. M., Parreira, F. J. \& Shitsuka, R. (2018). Metodologia da pesquisa científica. UFSM.

Pimentel, E. F., Dias, R. S., Ribeiro-Cunha, M., \& Glória, M. B. A. (2002). Avaliação da rotulagem e da qualidade físico-química e microbiológica de queijo ralado. Ciência e Tecnologia de Alimentos, 22(3). https://doi.org/10.1590/S0101-20612002000300016

Pinto, A. T., Schmidt, V., Raimundo, S. A., \& Raihmer, F. (2007). Uso de ozônio no controle de fungos em sala de maturação de queijos. Acta Scientiae Veterinariae, 35(3), 333. https://doi.org/10.22456/1679-9216.16125

Sora, G. T. de S., Franciosi, A. E. S., Bergamasco, R., \& Vieira, A. M. S. (2013). Development and the physical, chemical, microbiological and sensory analyses of red pepper seasoned with parmesan cheese. Acta Scientiarum. Technology, 35(3). https://doi.org/10.4025/actascitechnol.v35i3.12794

Srey, S., Jahid, I. K., \& Ha, S.-D. (2013). Biofilm formation in food industries: A food safety concern. Food Control, 31(2), 572-585. https://doi.org/10.1016/j.foodcont.2012.12.001

Starikoff, K. R., Fontanesi, C. D., Maciel, F. M., Ikuta, C. Y., Ferreira, F., Ferreira Neto, J. S., Dias, R. A., Amaku, M., Cortez, A., Heinemann, M. B., GrisiFilho, J. H. H., Gonçalves, V. S. P., Silva, P. H. F. da, Paula, J. C. J. de, \& Telles, E. O. (2016). Decline in Mycobacterium bovis and Brucella abortus populations during the maturation of experimentally contaminated parmesan-type cheese. Semina: Ciências Agrárias, 37(5Supl2), 3743. https://doi.org/10.5433/16790359.2016v37n5Supl2p3743

Trombete, F. M., Castro, I. M. de, Teixeira, A. da S., Saldanha, T., \& Fraga, M. E. (2013). Aflatoxin M1 contamination in grated parmesan cheese marketed in Rio de Janeiro - Brazil. Brazilian Archives of Biology and Technology, 57(2), 269-273. https://doi.org/10.1590/S1516-89132013005000015 